\title{
Craniovertebral junction reducible and irreducible compressive pathologies: free hands or free tools? Light and shadows in paediatric practice
}

\author{
Massimiliano Visocchi ${ }^{1}$ \\ Published online: 5 May 2020 \\ (C) Springer-Verlag GmbH Germany, part of Springer Nature 2020
}

In this paper titled Atlantoaxial instability treated with freehand C1-2 fusion in a 3-year old boy with Morquio syndrome, the authors, Eun Ji Moon, MD, Sang Joon Chong, MD, PhD and Jin Hoon Park, MD, PhD describe the case of a just 3-year Morquio old boy presenting with a 5-month history of progressive quadriparesis and severe neck pain [1]. The preoperative cervical imaging showed a compressive myelopathy at C1-2 and atlantoaxial subluxation. Atlantoaxial fixation and decompression were performed successfully with posterior C1-2 fusion using a free-hand technique. After the operation, the patient had improved neck pain and quadriparesis, and finally, he had been able to walk independently after postoperative 8 months. The authors conclude that this is the first report dealing with a paediatric Morquio patient treated just with C1-2 screwing and fusion with a free-hand technique. As far as I realize, the most important topic of this paper is the biochemical considerations on Morquio syndrome, the odontoid malformations, the surgical options and the intraoperative assistance.

\section{Biochemical considerations}

In this special case, the clinical issue is carried by Morquio syndrome, also known as Mucopolysaccharidosis type IV (MPS IV); as well known, this is a rare metabolic disorder in which the body cannot process certain types of sugar molecules called glycosaminoglycans (AKA GAGs or mucopolysaccharides). In Morquio patients, the specific GAG which builds up in the body is called keratan sulfate. This birth

Massimiliano Visocchi

mvisocchi@hotmail.com

1 Craniovertebral Junction Operative Unit, Fondazione Policlinico Universitario A. Gemelli, Institute of Neurosurgery, Catholic University of Rome, Rome, Italy defect, which is autosomal recessive, is a type of lysosomal storage disorder. The build-up of GAGs in different parts of the body, along with developmental anomalies, causes symptoms in many different organ systems. In the USA, the incidence rate for Morquio is estimated at between 1 in 200,000 and 1 in 300,000 live births [2,3].

\section{Odontoid malformations}

A whole body skeletal survey in Morquio syndrome generally shows skeletal dysplasia with hypoplasia, thoracolumbar kyphosis and dysmorphic, flattened vertebral bodies with short stature, odontoid hypoplasia and atlanto-axial instability are pathognomonic features of Morquio syndrome. Historically, atlantoaxial instability with skeletal dysplasia has been treated by occipitocervical fusion with external orthosis $[2,3]$. Considering these patients' bone size, quality and age in these genetic disorders of skeletal dysplasia, C1-2 fusion is hard to establish stability. For sure, the experience of the Korean neurosurgeons is innovative and represents a new paradigm in this surgery.

In Morquio syndrome os odontoideum, not always ossified, is present in every case and the ring of atlas tends to be narrowed and thickened. Although $\mathrm{C} 1-\mathrm{C} 2$ instability is mild, severe spinal cord compression can occur because of both anterior extradural soft tissue thickening and invagination of the posterior arch of $\mathrm{C} 1$ into the foramen magnum due to condylar hypoplasia. According to Crockard, every Morquio patient should be investigated for spinal cord compression between the ages of 3 and 8 years. Treatment aims to avoid neurological damage or at least to arrest the progression of neurological disability. In cases of Morquio patients harbouring a demonstrated reducible os odontoideum instability, surgical reduction appears mandatory; according to Crockard's suggestion, surgery appears to prevent 
neurological damage. Otherwise a transoral or transnasal decompression and posterior fusion can be combined in cases with significant anterior compression $[2,3]$.

When dealing with odontoid malformations, it is necessary to differentiate ossiculum terminale, os odontoideum and os avis, according to the most updated literature [1]. Malformations of the dens include aplasia, hypoplasia, duplication, condylus tertius, os terminale and os odontoideum. Among the most important features, Os odontoideum (plural: ossa odontoidea) is an anatomic variant of the odontoid process of $\mathrm{C} 2$ and needs to be differentiated from persistent ossiculum terminale and from a type 2 odontoid fracture. It can be associated with atlantoaxial instability. Although it was originally thought to be a congenital lesion due to a failure of the centre of ossification of the dens to fuse with the body of $\mathrm{C} 2$, it may actually represent an unremembered and/or unrecognised fracture through the dens' growth plate (synchondral fracture) before the age of 5 or 6 . There may be associated instability and chronic symptoms The level of mobility is below the transverse band of the cruciform ligament and therefore results in abnormal mobility of the dens with respect to $\mathrm{C} 2$. In other words, atlantoaxial instability is more common with os odontoideum. An os odontoideum can be divided into two main types 2: orthotopic, normal position with a wide gap between $\mathrm{C} 2$ and os odontoideum dystopic, displaced (dystopic os odontoideum has also been termed the 'os avis' by some) Association Morquio syndrome, Down Syndrome and multiple epiphyseal dysplasia. It may be seen in association with another adjacent anatomic variant, the third condyle Radiographic features smooth, well-corticated ossicle at the superior ossicle of a hypoplastic dens, around half the size of a normal dens associated with hypertrophied and rounded anterior arch of the atlas [2].

Ossiculum terminale Ossiculum terminale (Os avis by some) appears as a secondary ossification centre of the dens between 3 and 6 years and normally fuses by 12 years. Failure of fusion results in a persistent ossiculum terminale (also called Bergmann's ossicle or ossiculum terminale of Bergmann) and is considered a normal anatomical variant of the axis. It lies above the alar ligament and is therefore considered to be stable, and it very rarely causes symptoms. Radiographic feature, small, well-corticated ossicle at the tip of the dens usually in the midline dens, is typically normal in height differential diagnosis os odontoideum, type 1 odontoid fracture [2].

The third condyle The third condyle also known as condylus tertius or median occipital condyle is a rare anatomic variant of the occipital condyles. It is a small separate ossicle at the anteromedial margin of the occipital condyle formed by the failure of the embryonic proatlas (the 4th occipital sclerotome) to unite with the condyle proper. It may form a true arthrosis or pseudoarthorosis with the adjacent odontoid process of the axis or anterior arch of the atlas and has an association with an os odontoideum. The third condyle represents a spectrum of occipital vertebrae variations, and the 3 other most common variants include basilar process, paracondylar process and prebasioccipital arch [2].

\section{Surgical options}

The intraoperative essentials in this special and challenging surgery are head traction, fluroscopic assistance and neurophysiological monitoring (motor and sensory evoked potentials). Surgical approach to the anterior craniovertebral junction (CVJ) (in this case Morquio syndrome) can concern anterior decompressive procedures i.e. endoscopic transnasal approach (EEA) and transoral approach (TOA) (the latter also microsurgical) in childhood. Moreover, the recently introduced transnasal endoscopic odontoidectomy accomplishes a good decompression of the bulbomedullary junction with $100 \%$ avoiding the need of the tracheostomy and sometimes also avoiding the need of posterior instrumentation and fusion in those cases with sparing of the transverse ligament [4-7].

Concerning posterior instrumentation procedures, one stage combined (with EEA and TOA) or stand alone, when dealing with reducible dislocations, screwing technique are gaining much more success, since screw-based constructs provide superior stabilization and biomechanical profiles when compared with sublaminar wires $[8,9]$. Otherwise, screwing constructs can be affected by radiotherapy and consequently may require to be converted in wiring ones; furthermore, also postoperative infections can weaken the construct so requiring the hardware removal but sometimes without the need to performing a reinstrumentation procedure due to the reactive secondary bone regrowth $[10,11]$.

In children younger than 3 years, surgical instrumentation may raise concerns due to a patient's age and desirability of maintaining the growth of an immature spine. In these cases, delicate and small bony structures may not withstand rigid instrumentation, due to excessive mobility, poor bone purchase at the time of instrumentation and still developing ossification centres with cartilaginous interpositions. So, within the cervical spine, the use of rods with absorbable versus non-absorbable sublaminar sutures has been matter of debate according to personal preference [3]. In our experience, the old fashion occipitocervical $(\mathrm{C} 0-\mathrm{C} 3)$ wiring instrumentation, performed on a 8 years old Morquio patient, allowed a good functional compensation with a quite normal CVJ movement pattern at 22-year follow-up. So, an 'extensive CVJ fixation' although considered an historical procedure seems to continue to deserve consideration. [3] (Figs. 1 and 2).

We cannot forget that some of the so-called irreducible $\mathrm{Cl}-$ $\mathrm{C} 2$ dislocations during dynamic X-Ray and in Halo traction can become reducible in the operating room, under general 


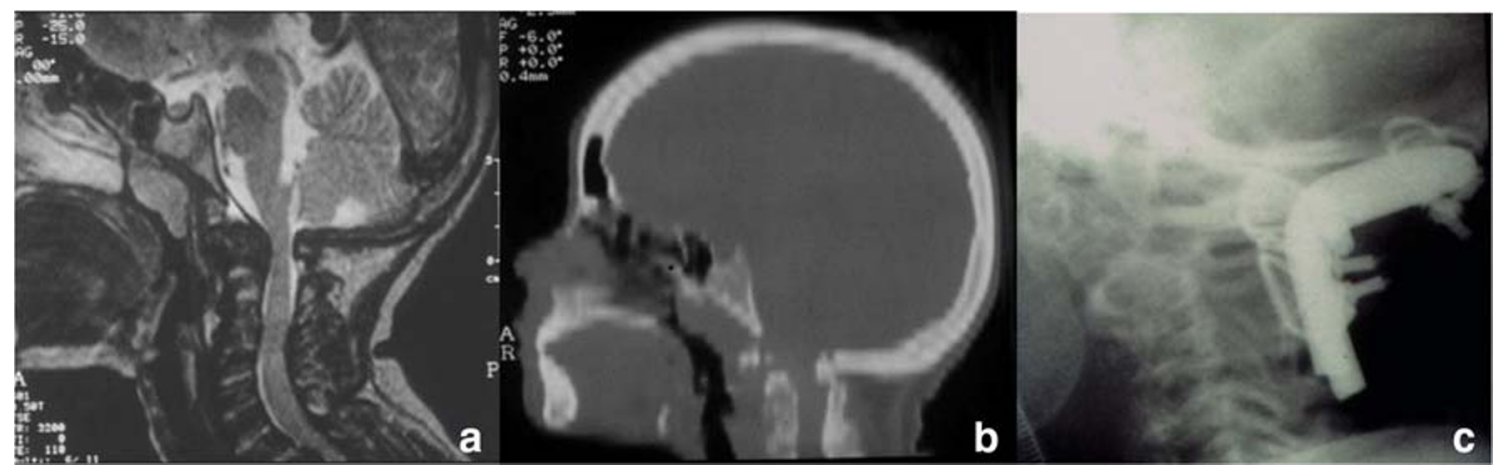

Fig. 1 Morquio patient operated 22 years ago (personal observation). Preoperative a MR T2 W and b CT scan sagittal reconstruction showing bulbomedullary junction compression and CVJ complex malformation $\mathbf{c}$ postoperative X-Ray control showing the $\mathrm{C} 0-\mathrm{C} 3$ wiring construct

anaesthesia, curarization, with prone position, with a simple head Mayfield traction, by using special lever intraoperative manoeuvres, both with wiring and screwing instrumentation techniques. Such an observation has reduced the number of anterior decompressive procedures. This philosophy, valid also in childhood and in Morquio patients, was published in 2009 with 'the always posterior strategy': in all the cases, a posterior attempt to reducing and fixating $\mathrm{C} 1-\mathrm{C} 2$ should be done $[7,8]$.

In the case published by Moon E et al., a fluoroscopicassisted screwing technique was performed without any CT neuronavigation (free-hand technique). Although I feel most attracted by the simpler intraoperative fluoroscopy in conventional surgical procedures, in this case, I should have strongly advised neuronavigation, due to the complexity of the local CVJ anatomy and the local severity of Morquio disease.

\section{Intraoperative assistance}

\section{Lights}

O-Arm or Intraoperative (preoperative) CT scan acquisition, comparing with fluoroscopy, not only have the obvious advantage of a better definition with a resulting easier screws insertion, but, for sure, it permits an intraoperative direct and indirect assessment of bony and ligamentous CVJ anterior decompression. In our experience with O-Arm acquisition, the cranio-caudal decompression can be augmented intraoperatively because of the possibility to correct the surgical technique when it is proved to be suboptimal. Also in TOA procedures, the use of Iopamire with fluoroscopy as contrast filler indirectly allows to evaluate possible residual compression at the CVJ, but it does not provide a real-time visualization of the decompression. In complex anatomies (CT/OArms), neuronavigation allows to better approach children in both anterior and posterior CVJ procedures.

\section{Shadows}

If required, the possibility to convert intraoperativeneuronavigated $3 \mathrm{D}$ modality into $2 \mathrm{D}$ real-time with O-Arm appears to be uncomfortable due to the poor space volume available for the surgeon and the need of complex, time consuming and often not effective surgical maneuvres required.

Therefore, sufficient experience in posterior CVJ complex surgery and confidence with $\mathrm{O}$ Arm/Intraoperative (preoperative) CT navigation is needed to safely perform this

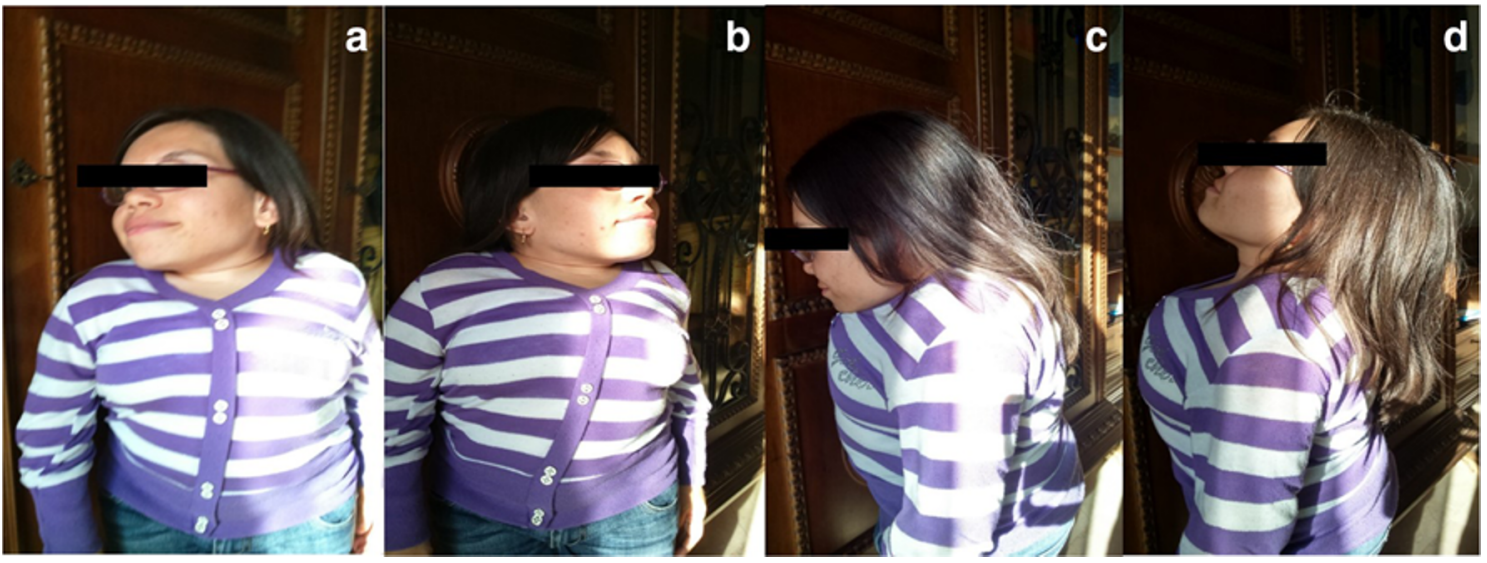

Fig. 2 Morquio patient operated 22 years ago (personal observation). a Dx rotation, $\mathbf{b}$ sx rotation, $\mathbf{c}$ flexion and $\mathbf{d}$ extension at maximum follow-up 
procedure, as demonstrated by a $1 \mathrm{~h}$ longer $(62 \mathrm{~min})$ mean operation time of our series compared with the personal previous data published (data submitted for publication).

\section{Conclusions}

Experience from our case series and a review of the literature support the need:

1) to make worldwide available and also economically accessible (free tools) the neuronavigation (intraoperative or preoperative)-assisted CVJ surgery mainly in paediatric surgery, since the bone fragility and incomplete local anatomic development mandate 'one shot surgery';

2) to spare, as much as possible, the local anatomy, the bone purchase and the local biomechanical potential;

3) to carefully select personalized occipital plates, rods, screws and wire constructs, depending on the underlying pathology, the bone thickness, age and degree of suspected instability. Thin or weak bone, revision surgeries with or without intraspinal pathologies, difficulty in compliance with immobilization and skin integrity secondary to proud instrumentation on the occiput remain challenging issues.

\section{References}

1. Moon E, Lee S, Chong S, Park JH (2020) Atlantoaxial instability treated with free-hand C1-C2 fusion in a child with Morquio syndrome. Childs Nerv Syst. https://doi.org/10.1007/s00381-02004561-2 PMID: 32172394

2. Visocchi M, Di Rocco C (2014) Os odontoideum syndrome: pathogenesis, clinical patterns and indication for surgical strategies in childhood. Adv Tech Stand Neurosurg 40:273-293. https://doi.org/ 10.1007/978-3-319-01065-6_9 PMID: 24265050
3. Visocchi M, Fernandez EM, Ciampini A, Di Rocco C (2009) Reducible and irreducible os odontoideum treated with posterior wiring, instrumentation and fusion. Past or present? Acta Neurochir (Wien) 151(10):1265-1274

4. Visocchi M (2016) Considerations on "endoscopic endonasal approach to the craniovertebnral junction: the importance of the anterior $\mathrm{C} 1$ arch preservation or its reconstruction". Letter to the editor. Acta Otorhinolaringol Ital 39:1-3. https://doi.org/10.14639/0392100X-927

5. Visocchi M, Della Pepa GM, Doglietto F, Esposito G, La Rocca G, Massimi L (2011) Video-assisted microsurgical transoral approach to the craniovertebral junction: personal experience in childhood. Childs Nerv Syst 27(5):825-831. https://doi.org/10.1007/s00381010-1386-5 PMID: 21240509

6. Visocchi M, Signorelli F, Liao C, Rigante M, Paludetti G, Barbagallo G, Olivi A (2017) Endoscopic endonasal approach for craniovertebral junction pathologic conditions: myth and truth in clinical series and personal experience. World Neurosurg 101:122129. https://doi.org/10.1016/j.wneu.2017.01.099 Review

7. Visocchi M, Signorelli F, Liao C, Rigante M, Paludetti G, Barbagallo G, Olivi A (2018) Transoral versus transnasal approach for craniovertebral junction pathologies: never say never. World Neurosurg 110:592-603. https://doi.org/10.1016/j.wneu.2017.05. 125

8. Visocchi M, Pietrini D, Tufo T, Fernandez E, Di Rocco C (2009) Pre-operative irreducible C1-C2 dislocations: intra-operative reduction and posterior fixation. The "always posterior strategy". Acta Neurochir 151(5):551-559. https://doi.org/10.1007/s00701-0090271-z discussion 560. PMID: 19337686

9. Visocchi M (2009) Response to Wang et al., re Letter re Visocchi M, Pietrini D, Tufo T, Fernandez E, Di Rocco C (2009) Preoperative irreducible $\mathrm{C} 1-\mathrm{C} 2$ dislocations: intra-operative reduction and posterior fixation. The "always posterior strategy". Acta Neurochir 151(5):551-559 discussion 560

10. Visocchi M, Sturiale C (2012) G Esposito: occipitocervical instrumentation failure after radio - chemio therapy for solitary axis plasmocytoma: a case - based update. Eur J Orthop Surg Traumatol 22(suppl):S45-S51

11. Visocchi M, Mattogno PP, Signorelli F, Zhong J, Iacopino G, Barbagallo G (2017) Complications in craniovertebral junction instrumentation: hardware removal can be associated with longlasting stability. Personal Experience. Acta Neurochir Suppl 124: 187-194. https://doi.org/10.1007/978-3-319-39546-3_29

Publisher's note Springer Nature remains neutral with regard to jurisdictional claims in published maps and institutional affiliations. 